\title{
Spreading-Resistance Temperature Sensor on SOI
}

\author{
P.T.Lai, Bin Li and C.L.Chan \\ Department of Electrical and Electronic Engineering, the University of Hong Kong \\ (E-Mail:laip@hkueee.hku.hk)
}

\begin{abstract}
A Spreading-Resistance Temperature (SRT) sensor is fabricated on silicon-oninsulator (SOI) wafer and achieves characteristics comparable with similar SRT sensor on silicon wafer. This sensor structure can be potentially used in integrated sensors operating at temperatures up to $350^{\circ} \mathrm{C}$.
\end{abstract}

\section{Introduction}

Conventional silicon temperature sensors are based on the principle that the resistivity of silicon increases with temperature in the region between $-50^{\circ} \mathrm{C}$ and $+150{ }^{\circ} \mathrm{C}$. Due to its low cost of processing, this kind of sensors is welcome by industrial manufacturers. However, the rather low maximum operating temperature of these silicon sensors prevents their implementation in a number of potential applications, especially in the automotive industry. To cope with this problem, SpreadingResistance Temperature (SRT) sensor was firstly reported by G. Raage in 1982 [1]. The main characteristic of SRT sensor is that it can operate in a wider temperature range from $-50{ }^{\circ} \mathrm{C}$ to around $+300{ }^{\circ} \mathrm{C}$. Hence, these sensors can be potentially used in a multitude of applications, where conventional silicon sensors are not suitable. The SRT sensor is based on the minority-carrier exclusion effect [2] at high-low junctions which suppresses the effect of thermal generation of carriers and maintains extrinsic-carrier concentrations at intrinsic temperatures, thereby increasing the maximum operating temperature of the device.

The SRT sensor has been around over 10 years $[1,3]$. Though it achieves good characteristics up to $300{ }^{\circ} \mathrm{C}$, its vertical structure has, to some extent, impeded a wider scope of applications. One of the breakthroughs of SRT sensor is the lateral structure proposed in [4]. As shown in Fig.1, the major advantage of the new SRT structure is that the two external connections of the device are on the same side of the silicon wafer. As a result, the

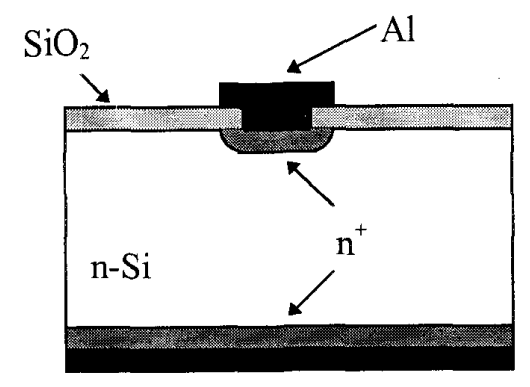

(a)

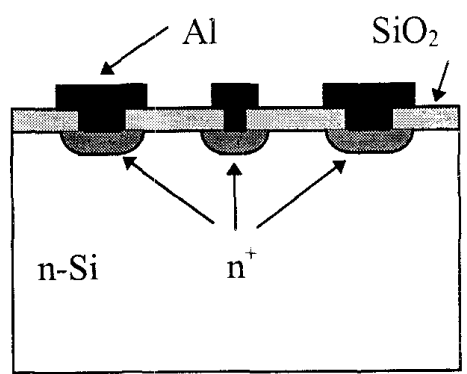

(b)

Fig. 1 (a) Cross-section of traditional SRT sensor. (b) Cross-section of novel SRT sensor. 
processing is compatible with the planar IC technology, and the device can be integrated with other devices or circuits on a small piece of silicon chip. Hence, the applications of this SRT sensor cover a much wider area. One application of this novel SRT sensor in integrated flow sensor has been reported [4-5]. However, with pn junctions as isolation between the SRT sensors, the chip cannot operate under ambient temperatures above $150{ }^{\circ} \mathrm{C}$ due to high leakage current of the junctions. In this paper, silicon on insulator (SOI) with mesa isolation is adopted to eliminate the pn junctions and SRT sensors are fabricated on the silicon islands of the SOI wafer. This new structure can also provide an extra dimension (silicon-film thickness) for optimising the characteristics of the sensor. Experimental results show that the maximum operating temperature of SOI SRT sensor can go up to $350^{\circ} \mathrm{C}$.

\section{Device Fabrication}

SRT sensors were fabricated on commercially available SOI wafers formed by the separation by implantation of oxygen (SIMOX) technique with an n-type $<100>$ siliconfilm thickness of $10 \mu \mathrm{m}$, and a buriedoxide thickness of $0.4 \mu \mathrm{m}$. Conventional bulk wafers were also processed by the same technology for comparison. The silicon film and the bulk wafer both had a resistivity of $10 \sim 20 \Omega . \mathrm{cm}$. The $\mathrm{n}^{+}$ regions in Fig. $1 \mathrm{~b}$ with a junction depth of $0.8 \mu \mathrm{m}$ for the SRT sensors were formed by thermal diffusion of phosphorus at $1000{ }^{\circ} \mathrm{C}$ for $15 \mathrm{~min}$. The sensor had a circular $\mathrm{n}^{+}$region (diameter is $20 \mu \mathrm{m}$ ) at the centre, with a ring $\mathrm{n}^{+}$ region (inner and outer diameters are 100 and $200 \mu \mathrm{m}$ respectively) surrounding it .

\section{Results and discussions}

The resistance of the device versus temperature is measured for the two types of SRT sensors (SOI and bulk) using a conventional temperaturecontrolled oven. Fig. 2 and Fig. 3 show the device resistance versus temperature at different bias currents for SOI and bulk SRT sensors respectively. In Fig.2, the maximum operating temperature of the SOI SRT sensor is over $300{ }^{\circ} \mathrm{C}$ and increasing the bias current in the forward direction (the circular $\mathrm{n}^{+}$region is at a higher potential than the ring $\mathrm{n}^{+}$region) can increase the maximum operating temperature. On the contrary, reversing the bias current cannot result in the minority exclusion effect, and so the maximum operating temperature is only $200{ }^{\circ} \mathrm{C}$, just like conventional resistor structure. This is in good agreement with the minority exclusion effect, which occurs in both SOI and bulk SRT sensors.

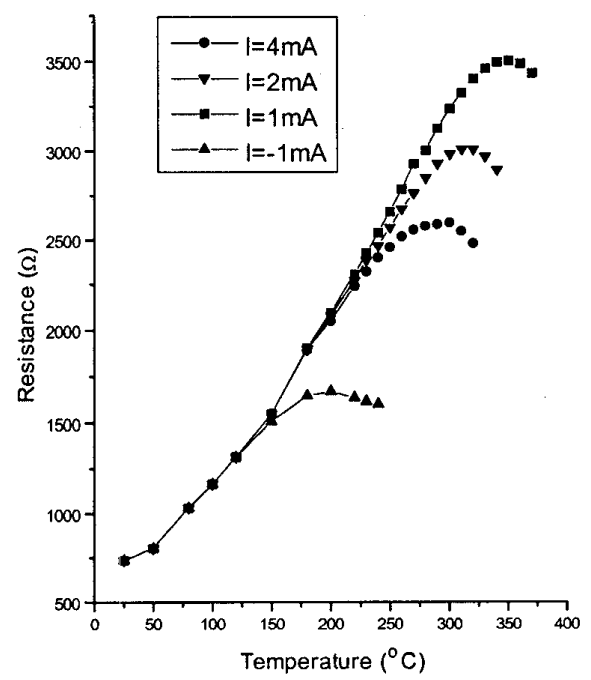

Fig.2 Temperature dependence of the resistance of SOI SRT sensor biased at different current levels. 


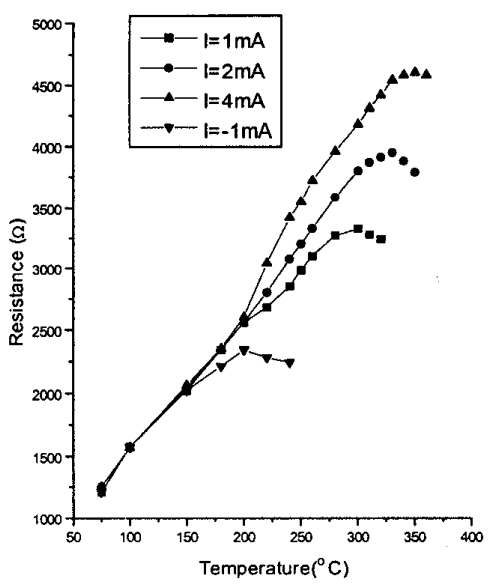

Fig.3 Temperature dependence of the resistance of bulk SRT sensor biased at different current levels.

Due to the specific structure of the SOI sensor, a bias voltage can be applied at its back which significantly affects the R-T characteristics of the sensors. Fig. 4 shows when the substrate is applied a negative bias voltage, the maximum operating temperature can be increased. The higher the negative substrate voltage, the higher is the maximum operating temperature. On the contrary, a positive substrate voltage hardly affects the maximum operating temperature, with only a decrease in the sensor resistance due to the formation of an accmulation layer above the siliconfilm/buried-oxide interface. On the other hand, as shown in Fig.5, when a negative substrate voltage is used, there is a depletion region in the silicon film extending upwards from the buried oxide, giving rise to a reduction of the silicon-film thickness. The resulting higher maximum operating temperature of the sensor is possibly due to the fact that the intrinsic portion of the sensor is reduced and thus the device resistance is less affected by thermal generation of charge carriers.

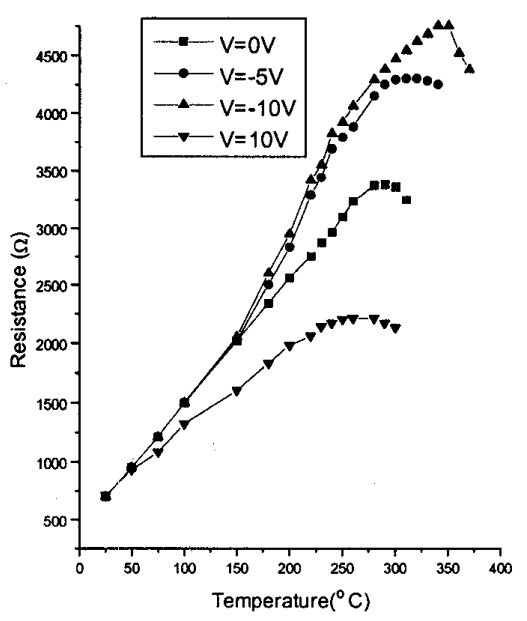

Fig.4 Temperature dependence of the resistance of SOI SRT sensor at different substrate bias voltages (Bias current $=$ $1 \mathrm{~mA})$

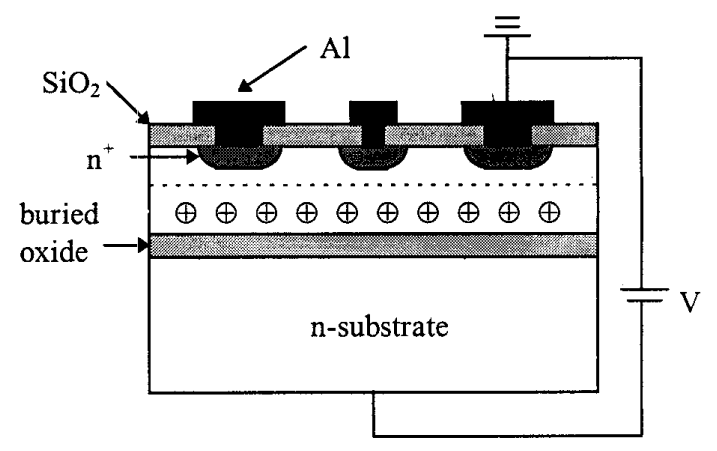

Fig.5 Effect of a negative voltage at the substrate on the charge distribution in the silicon film

\section{Conclusions}

Preliminary results support the feasibility of making SRT sensors on SOI materials. The characteristics of the SOI SRT sensor compare favourably with those of SRT sensor fabricated on bulk wafer. 


\section{References}

[1]G.Raabe, Silizium-temperaturesensoren von $-50^{\circ} \mathrm{C}$ bis $+350^{\circ} \mathrm{C}, N T G$ Fachberichte, 79 (1982) 248-253.

[2] G.G.E. Low, Carrier concentration disturbances in semiconductors, Proc. Phys. Soc. B, 68 (1955)310-314.

[3] S.R. in't Hout and S. Middelhoek, $400^{\circ} \mathrm{C}$ silicon Hall sensor, Sensors and Actuators A,60(1997) 14-22.

[4] P.T.Lai and Baiyong Liu, Monolithic integrated spreading-resistance silicon flow sensor, Sensors and Actuators A,58 (1997) 85-88.

[5] P.T.Lai and Baiyong Liu, A twodimensional flow sensor using integrated silicon spreading-resistance temperature detectors, Rev. Sci. Instrum., 68(1997) 10-15. 